\title{
Palatal Rugae Pattern in Korean Children and Adolescents
}

\author{
Na-Hee Kim¹, Yeong-Gwan Im¹, Ji-Yeon Kim², Byung-Gook Kim¹ \\ ${ }^{1}$ Department of Oral Medicine, School of Dentistry, Chonnam National University, Gwangju, Korea \\ ${ }^{2}$ Department of Oral Medicine, Seoul Veterans Hospital, Seoul, Korea
}

Received November 26, 2019

Revised December 19, 2019

Accepted December 20, 2019
Correspondence to:

Byung-Gook Kim

Department of Oral Medicine, School of

Dentistry, Chonnam National University, 33

Yongbong-ro, Buk-gu, Gwangju 61186,

Korea

Tel: +82-62-530-5574

Fax: +82-62-530-5679

E-mail: bkkim@jnu.ac.kr

https://orcid.org/0000-0002-3602-4720
Purpose: To determine whether the morphological features of the palatal rugae are associated with sex and age in children and adolescents.

Methods: A total of 300 diagnostic models of the palatal rugae of children and adolescents were collected. The models were classified into male and female and $<13$ - and $\geq 13$-year-old groups. The palatal rugae pattern, and the number and length of palatal rugae plicae, were analyzed.

Results: The number of palatal rugae plicae was higher in females than in males, however, the difference was negligible. In the group aged 13 years or more, the number was higher in the male group on the left side. There was no association between the number of palatal rugae plicae and age group. The type I pattern was the most common in both males and females. The length of palatal rugae plicae was greater in males than in females. There was no association between the length of palatal rugae plicae and age group.

Conclusions: The number and length of palatal rugae were associated with sex, but the morphological features of the palatal rugae could not distinguish between children and adolescents. These findings suggest that the palatal rugae have limited value for identification of individuals.

Key Words: Forensic dentistry; Individual identification; Palatal rugae; Palate

\section{INTRODUCTION}

Various types of disasters, including structural collapses, fires, and explosions, as well as accidents involving trains, ships, and aircraft, can result in large numbers of casualties. Forensic identification of individuals is required when such disasters occur and cause many deaths. The identification of individuals in this context involves the examination of both living and dead bodies. Many methods for human identification have been developed. In 1932, Hermosa et al. introduced palatoscopy based on palatal rugae patterns for human identification, which can be applied even if the teeth are severely damaged [1].

The palatal rugae are anatomical ridges with asymmetric and irregular shapes located in the anterior one-third of the palate. They are formed between 12 and 14 weeks of birth, and extend outward on both sides from the palatal closure $[2,3]$. Thomas [4] studied the histologic features of rugae cores from human embryos aged over 20 weeks and found that the reticulin fiber content was miniscule; moreover, the fibroblasts differed from those in adjacent palatal tissues only in size and amount. The palatal rugae can be used to identify a dead body because of their specificity and stability [5]. Moreover, the palatal rugae can be used as an alternative method when individual identification based on fingerprints or dental records is difficult, or when postmortem dental identification is impossible, as in cases with edentulous mouths [6].

Many studies have attempted to use the palatal rugae for human identification [7]. Thomas and Kotze [8] studied the

Copyright (c) 2019 Korean Academy of Orofacial Pain and Oral Medicine. All rights reserved.

(c) This is an open-access article distributed under the terms of the Creative Commons Attribution Non-Commercial License (http://creativecommons.org/licenses/by-nc/4.0/), which permits unrestricted non-commercial use, distribution, and reproduction in any medium, provided the original work is properly cited. 
pattern of palatal rugae plicae of six South African populations and found that the palatal rugae are unique and can be used for genetic research. Chae and Shin [9] reported individuality of palatal rugae pattern in twin children and adolescents. However, although prior studies used palatal rugae data for individuals of all ages, they did not specifically evaluate the effect of age on the palatal rugae [8].

We investigated the morphological features of the palatal rugae of children and adolescents, and examined the association between those features and age. Additionally, we explored the possibility of using palatal rugoscopy for identification of individual children and adolescents.

\section{MATERIALS AND METHODS}

\section{Subjects}

Diagnostic models of adolescent male and female subjects aged 5 to 18 years were obtained from the Department of Pediatric Dentistry of Chonnam National University Dental Hospital. Diagnostic models with intact palatal rugae patterns were selected and divided into male and female groups. The models were further divided into children $(<13$ years old) and adolescents ( $\geq 13$ years old) (Table 1).

\section{Analysis of Palatal Rugae}

The plicae of the palatal rugae of each diagnostic model were sketched using a sharp mechanical pencil. Photographs were obtained with a standard ruler placed adjacent to the models at a standardized distance [10]. The patterns, numbers, and lengths of palatal rugae plicae were analyzed. Palatal rugoscopy was based on the methods developed by Thomas and Kotze [8] and Lysell [11]. The palatal rugae pattern was classified into the following four types: type I, straight; type II, branched; type III, circular; and type IV, mixed (Fig. 1) [10,12].

Table 1. Distribution of the subjects according to age group and sex

\begin{tabular}{cccc}
\hline Age group & Male & Female & Total \\
\hline$<13 y$ & 122 & 117 & $239(79.7)$ \\
$\geq 13 y$ & 38 & 23 & $61(20.3)$ \\
Total & $160(53.3)$ & $140(46.7)$ & $300(100.0)$ \\
\hline
\end{tabular}

Values are presented as number only, number (\%).

\section{Statistical Analysis}

The Pearson chi-squared test was used to compare the palatal rugae pattern distributions between the male and female groups. The independent t-test was used to compare the number and length of the palatal rugae plicae, and to examine differences between the two age groups. Statistical analyses were performed using IBM SPSS Statistics for Windows, Version 21.0 (IBM Co., Armonk, NY, USA). Ethics approval was obtained from the Institutional Review Board of Chonnam National University Dental Hospital (IRB no. CNUDH-2019-002).

\section{RESULTS}

\section{Number of Palatal Rugae Plicae}

The mean number of palatal rugae plicae on the left and right sides of all age groups ranged from 3.2 to 3.4. The difference in number of palatal rugae plicae between males and females was significant on both the left $(p<0.05)$ and the right $(\mathrm{p}<0.05)$ side. Although the mean number was higher in the female group on both sides, the difference was only between 0.1 and 0.2 (Table 2). In the group aged less than 13 years, the difference was significant both on the left $(\mathrm{p}<0.05)$ and the right $(\mathrm{p}<0.05)$ side (Table 3$)$. In the group aged 13 years or more, however, the number

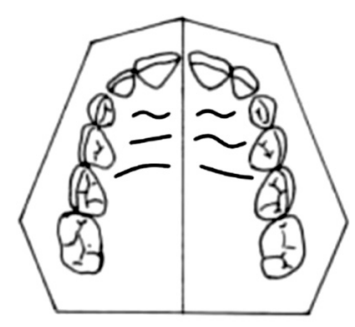

Type I: straight

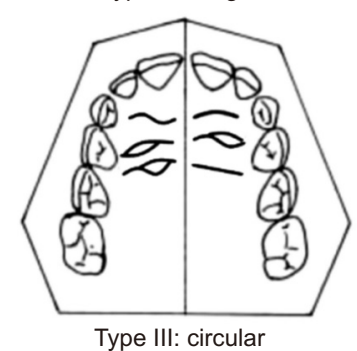

Type III: circular

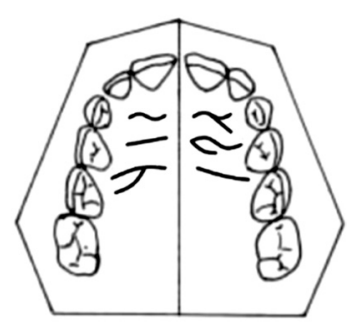

Type II: branched

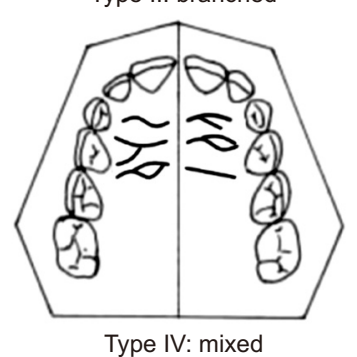

Fig. 1. Classification of the types of palatal rugae pattern based on the methods developed by the article of Surekha et al. (J Forensic Dent Sci 2012;4:93-96) [10] and Hauser et al. (J Anat 1989;165:237249) [12]. 
was significantly higher in the male group on the left side $(p<0.05)$ (Table 3). A comparison of the number of palatal rugae plicae between the two age groups showed no significant difference, except in the female group on the left side $(\mathrm{p}<0.05)$ (Table 4).

\section{Distribution of the four types of palatal rugae pattern}

The most frequent type of palatal rugae pattern among all subjects $(n=300)$ was type $I$, which was found in 135 of

Table 2. Number of palatal rugae plicae according to sex

\begin{tabular}{lccc}
\hline Side & Male $(n=160)$ & Female $(n=140)$ & $p$-value \\
\hline Left & $3.2 \pm 0.7$ & $3.4 \pm 0.7$ & $<0.05$ \\
Right & $3.2 \pm 0.7$ & $3.3 \pm 0.6$ & $<0.05$ \\
\hline
\end{tabular}

Values are presented as mean \pm standard deviation.

$\mathrm{p}$-values were determined by independent t-test.

Table 3. Number of palatal rugae plicae according to sex and age group

\begin{tabular}{clccc}
\hline Age group & Side & Male & Female & p-value \\
\hline$<13 \mathrm{y}$ & Left & $3.2 \pm 0.7$ & $3.3 \pm 0.7$ & $<0.05$ \\
& Right & $3.2 \pm 0.7$ & $3.4 \pm 0.6$ & $<0.05$ \\
$\geq 13 \mathrm{y}$ & Left & $3.2 \pm 0.6$ & $3.0 \pm 0.7$ & $<0.05$ \\
& Right & $3.2 \pm 0.6$ & $3.2 \pm 0.6$ & NS \\
\hline
\end{tabular}

NS, not significant.

Values are presented as mean \pm standard deviation.

$\mathrm{p}$-values were determined by independent t-test.
300 subjects (45.0\%), followed by type IV (20.3\%), type II (19.0\%), and type III (15.7\%). For the male subjects, type I was the most common pattern, followed by type II, type IV, and type III, in order. For the female subjects, the order was type I, followed by types III, IV, and II; however, the difference in proportions of the four types of palatal rugae pattern were not significantly different $(p=5.465)$ (Table 5, Fig. 2).

The proportions of the four types of palatal rugae pattern were similar in the group aged less than 13 years $(p=3.867)$ (Table 6). Similar results were obtained in the group aged 13 years old or higher.

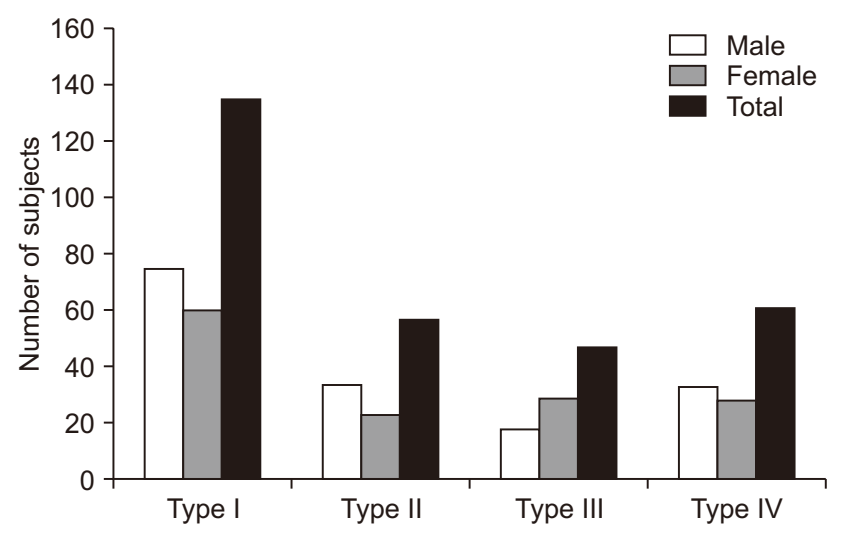

Fig. 2. Distribution of the four types of palatal rugae pattern.

Table 4. Number of palatal rugae plicae in the two age groups

\begin{tabular}{clllc}
\hline Sex & Side & $<13 y$ & $\geq 13 y$ & $\mathrm{p}$-value \\
\hline Male & Left & $3.2 \pm 0.7$ & $3.2 \pm 0.6$ & NS \\
& Right & $3.2 \pm 0.7$ & $3.2 \pm 0.6$ & NS \\
Female & Left & $3.3 \pm 0.7$ & $3.0 \pm 0.7$ & $<0.05$ \\
& Right & $3.4 \pm 0.6$ & $3.2 \pm 0.6$ & NS \\
\hline
\end{tabular}

NS, not significant.

Values are presented as mean \pm standard deviation.

$\mathrm{p}$-values were determined by independent t-test.

Table 5. Distribution of the four types of palatal rugae pattern $(n=300)$

\begin{tabular}{|c|c|c|c|c|c|c|}
\hline \multirow{2}{*}{ Sex } & \multicolumn{4}{|c|}{ Type of palatal rugae pattern } & \multirow{2}{*}{ Total } & \multirow{2}{*}{$p$-value } \\
\hline & Type I & Type II & Type III & Type IV & & \\
\hline Male & $75(46.9)$ & $34(21.3)$ & $18(11.3)$ & $33(20.6)$ & $160(100.0)$ & $5.465^{*}$ \\
\hline Female & $60(42.9)$ & $23(16.4)$ & $29(20.7)$ & $28(20.0)$ & $140(100.0)$ & \\
\hline Total & $135(45.0)$ & $57(19.0)$ & $47(15.7)$ & $61(20.3)$ & $300(100.0)$ & \\
\hline
\end{tabular}

Type I, straight; type II, branched; type III, circular; type IV, mixed.

Values are presented as number (\%).

${ }^{*} \mathrm{p}$-values were determined by the $\chi^{2}$ test. 
Table 6. Distribution of the four types of palatal rugae pattern in the group aged less than 13 years $(n=239)$

\begin{tabular}{|c|c|c|c|c|c|c|}
\hline \multirow{2}{*}{ Sex } & \multicolumn{4}{|c|}{ Type of palatal rugae pattern } & \multirow{2}{*}{ Total } & \multirow{2}{*}{ p-value } \\
\hline & Type I & Type II & Type III & Type IV & & \\
\hline Male & $60(49.2)$ & $24(19.7)$ & $13(10.7)$ & $25(20.5)$ & $122(100.0)$ & $3.867^{*}$ \\
\hline Female & $50(42.7)$ & $21(17.9)$ & $23(19.7)$ & $23(19.7)$ & $117(100.0)$ & \\
\hline Total & $110(46.0)$ & 45 (18.8) & $36(15.1)$ & $48(20.1)$ & $239(100.0)$ & \\
\hline
\end{tabular}

Type I, straight; type II, branched; type III, circular; type IV, mixed.

Values are presented as number (\%).

${ }^{*} \mathrm{p}$-values were determined by the $\chi^{2}$ test.

Table 7. Length of the palatal rugae plicae according to sex

\begin{tabular}{lccc}
\hline Side & Male $(n=160)$ & Female $(n=140)$ & $p$-value \\
\hline Left & $8.0 \pm 1.5$ & $7.4 \pm 1.4$ & $<0.05$ \\
Right & $8.6 \pm 1.6$ & $8.0 \pm 1.4$ & $<0.05$ \\
\hline
\end{tabular}

Values are presented as mean \pm standard deviation.

Unit of length, $\mathrm{mm}$.

$\mathrm{p}$-values were determined by independent t-test.

Table 8. Length of the palatal rugae plicae according to sex and age group

\begin{tabular}{clccc}
\hline Age group & Side & Male & Female & p-value \\
\hline$<13 y$ & Left & $7.8 \pm 1.5$ & $7.5 \pm 1.4$ & $<0.05$ \\
& Right & $8.5 \pm 1.5$ & $8.1 \pm 1.4$ & $<0.05$ \\
$\geq 13 y$ & Left & $8.3 \pm 1.5$ & $7.0 \pm 1.6$ & $<0.05$ \\
& Right & $8.8 \pm 1.8$ & $7.8 \pm 1.6$ & $<0.05$ \\
\hline
\end{tabular}

Values are presented as mean \pm standard deviation.

Unit of length, $\mathrm{mm}$.

$\mathrm{p}$-values were determined by independent t-test.

\section{Length of the Palatal Rugae Plicae According to Sex}

The mean length of the palatal rugae plicae ranged from 7.4 to $8.6 \mathrm{~mm}$. The length was greater in the male group on both the left and right sides. The difference in length between the two sex groups was significant on the left $(p<0.05)$ and the right $(\mathrm{p}<0.05)$ (Table 7) side. The mean length was greater in the male group on both sides among those aged less than 13 years, and among those aged 13 years or more (Table 8). There was no significant difference in the length of the palatal rugae plicae between the two age groups (Table 9).

\section{DISCUSSION}

The aim of this study was to determine whether the morphological features of the palatal rugae are associated with sex and age in children and adolescents.

Analysis of the palatal rugae has been used to aid
Table 9. Length of the palatal rugae plicae in the two age groups

\begin{tabular}{clccc}
\hline Sex & Side & $<13 y$ & $\geq 13 y$ & $p$-value \\
\hline \multirow{2}{*}{ Male } & Left & $7.8 \pm 1.5$ & $8.3 \pm 1.5$ & NS \\
& Right & $8.5 \pm 1.5$ & $8.8 \pm 1.8$ & NS \\
Female & Left & $7.5 \pm 1.4$ & $7.0 \pm 1.6$ & NS \\
& Right & $8.1 \pm 1.4$ & $7.8 \pm 1.6$ & NS \\
\hline
\end{tabular}

NS, not significant.

Values are presented as mean \pm standard deviation.

Unit of length, $\mathrm{mm}$.

$\mathrm{p}$-values were determined by independent t-test.

identification of individuals in the field of forensics. Most previous studies focused on the individual uniqueness of the palatal rugae. Hauser et al. [12] found that the shape and length of the palatal rugae of the palate were unchanged between birth and 9 years of age. Moreover, van der Linden [13] reported that the length of the palatal rugae did not change after 10 years of age, and their shape, orientation, and other qualitative characteristics also remained stable throughout the lifetime. According to Carrea [14], the palatal rugae remain unchanged for life, and orthodontic treatments and tooth loss do not influence their form. These findings illustrate the stability of the palatal rugae during developmental growth.

Indira et al. [15], Bing et al. [16], and Hermosilla et al. [17] found that males have a larger number of palatal rugae than females. In this study, the number of palatal rugae plicae was significantly higher in female than male subjects. However, the difference in absolute measurements between the two sexes was negligible; mean values were similar. We also found no difference in the number of palatal rugae plicae between children and adolescent subjects. It is thought that the number of palatal rugae plicae does not change throughout childhood and adolescence.

Hauser et al. [12] found a clear difference between Swazi 
and Greek populations in the pattern of the plicae palatine. In our study, type I was the most common pattern in both male and female subjects, and the distribution of the types was not significantly different between the sexes. This suggests that the type of palatal rugae pattern cannot be used for distinguishing between the sexes.

Lysell [11] reported that between the ages of 5 and 10 years, the length of the palatal rugae in the palate increases by $11 \%$ in males and $9 \%$ in females. In the present study, male subjects had longer palatal rugae plicae than female subjects. There was no difference in the length of the palatal rugae plicae between the children and adolescent subjects.

One major shortcoming of this study was that it involved only children and adolescents. Future studies should include a broader age range and larger numbers of children and adolescents, to verify our findings.

In conclusion, in general, palatal rugoscopy has limited value and cannot be considered a useful method for identifying individuals. However, some characteristics of the palatal rugae, such as their number and length of plicae, may be useful for distinguishing between the sexes.

\section{CONFLICT OF INTEREST}

No potential conflict of interest relevant to this article was reported.

\section{ORCID}

\author{
Na-Hee Kim \\ https://orcid.org/0000-0002-1254-2240 \\ Yeong-Gwan Im \\ https://orcid.org/0000-0003-2703-1475 \\ Ji-Yeon Kim \\ https://orcid.org/0000-0001-9127-096X \\ Byung-Gook Kim \\ https://orcid.org/0000-0002-3602-4720
}

\section{REFERENCES}

1. Paliwal A, Wanjari S, Parwani R. Palatal rugoscopy: establishing identity. J Forensic Dent Sci 2010;2:27-31.

2. Caldas IM, Magalhães T, Afonso A. Establishing identity using cheiloscopy and palatoscopy. Forensic Sci Int 2007;165:1-9.

3. Patil MS, Patil SB, Acharya AB. Palatine rugae and their significance in clinical dentistry: a review of the literature. J Am Dent Assoc 2008;139:1471-1478.

4. Thomas CJ. The prenatal developmental microscopic anatomy of the palatal rugae. J Dent Assoc S Afr 1984;39:527-533.

5. Jain A, Chowdhary R. Palatal rugae and their role in forensic odontology. J Investig Clin Dent 2014;5:171-178.

6. Thomas CJ. The role of the denture in identification: a review. J Forensic Odontostomatol 1984;2:13-16.

7. Ahn Y, Ko C, Huh K, et al. Consideration to the role of forensic odontology in determination of unidentified human body. J Oral Med Pain 2010;35:111-117.

8. Thomas CJ, Kotze TJ. The palatal ruga pattern: a new classification. J Dent Assoc S Afr 1983;38:153-157.

9. Chae G, Shin K. Individuality and stability of the palatal rugae pattern. J Oral Med Pain 2002;27:285-307.

10. Surekha R, Anila K, Reddy VS, Hunasgi S, Ravikumar S, Ramesh N. Assessment of palatal rugae patterns in Manipuri and Kerala population. J Forensic Dent Sci 2012;4:93-96.

11. Lysell L. Plicae palatinae transversae and papilla incisiva in man; a morphologic and genetic study. Acta Odontol Scand 1955;13(Suppl. 18):5-137.

12. Hauser G, Daponte A, Roberts MJ. Palatal rugae. J Anat 1989; 165:237-249.

13. van der Linden FP. Changes in the position of posterior teeth in relation to ruga points. Am J Orthod 1978;74:142-161.

14. Carrea JU. La Identificacion humana por las rugosidades palatinas. Rev Orthodont (Buenos Aires) 1937;1:3-23.

15. Indira A, Gupta M, David MP. Usefullness of palatal rugae patterns in establishing identity: preliminary results from Bengaluru city, India. J Forensic Dent Sci 2012;4:2-5.

16. Bing L, Wu XP, Feng Y, Wang YJ, Liu HC. Palatal rugae for the construction of forensic identification. Int J Morphol 2014;32: 546-550.

17. Hermosilla Venegas V, San Pedro Valenzuela J, Cantín López M, Suazo Galdames IC. Palatal rugae: systematic analysis of its shape and dimensions for use in human identification. Int J Morphol 2009;27:819-825. 